EPJ Web of Conferences 62, 05005 (2013)

DOI: $10.1051 /$ epjconf/20136205005

(C) Owned by the authors, published by EDP Sciences, 2013

\title{
FALSTAFF: A new tool for fission studies
}

\author{
D. Dore ${ }^{1, \mathrm{a}}$, F. Farget ${ }^{2}$, F.-R. Lecolley ${ }^{3}$, G. Lehaut ${ }^{3}$, T. Materna ${ }^{1}$, J. Pancin ${ }^{2}$, S. Panebianco ${ }^{1}$, \\ and Th. Papaevangelou ${ }^{1}$ \\ ${ }^{1}$ CEA, Centre de Saclay, Irfu/Service de Physique Nucléaire, 91191 Gif-sur-Yvette, France \\ ${ }^{2}$ GANIL, CEA/DSM-CNRS/IN2P3, Bd. H. Becquerel, 14076 Caen, France \\ ${ }^{3}$ LPC Caen, ENSICAEN, Université de Caen, CNRS-IN2P3, 14050 Caen Cedex, France
}

\begin{abstract}
The future NFS installation will produce high intensity neutron beams from hundreds of $\mathrm{keV}$ up to $40 \mathrm{MeV}$. Taking advantage of this facility, data of particular interest for the nuclear community in view of the development of the fast reactor technology will be measured. The development of an experimental setup called FALSTAFF for a full characterization of actinide fission fragments has been undertaken. Fission fragment isotopic yields and associated neutron multiplicities will be measured as a function of the neutron energy. Based on time-of-flight and residual energy technique, the setup will allow the simultaneous measurement of the complementary fragments velocity and energy. The performances of TOF detectors of FALSTAFF will be presented and expected resolutions for fragment masses and neutron multiplicities, based on realistic simulations, will be shown.
\end{abstract}

\section{Introduction}

The advent of the Neutrons For Science (NFS) facility at Caen (France) in 2015 will allow exploring the underexploited fast energy domain in order to provide data for fundamental physics and nuclear technology. On one hand, a lot of information is needed for the development of physics models to reach a complete and precise description of the fission process. On the other hand, the development of fast reactor technology and of reactors dedicated to the incineration of minor actinides shed light on the need of new nuclear data. These arguments have motivated the development of a new detection system called FALSTAFF (Four Arm cLover for the STudy of Actinide Fission Fragments). Based on timeof-flight (TOF) and residual energy technique, FALSTAFF will allow the simultaneous measurement of the complementary fragments nuclear charge, mass and kinetic energy. The $2 \mathrm{~V}$ and EV methods [1] will be used to determine the fragment mass before (initial mass $A_{i}$ ) and after (final mass $A_{f}$ ) neutron evaporation. The $2 \mathrm{~V}$ method based on mass conservation (assuming that the average fragment velocity is not changed by the evaporation process) allows to determine the fragment mass before neutron evaporation from the two fragments velocity which is measured by their time-of-flight. The EV method permits to identify the mass after neutron evaporation from a coincidence measurement

\footnotetext{
${ }^{\mathrm{a} e-m a i l: ~ d i a n e . d o r e @ c e a . f r}$
}

This is an Open Access article distributed under the terms of the Creative Commons Attribution License 2.0, which permits unrestricted use, distribution, and reproduction in any medium, provided the original work is properly cited. 
of energy and velocity. Neutron multiplicity as a function of the fragment mass may then be deduced directly from the difference between initial and final mass. The experimental setup and results obtained for time and spatial resolutions of TOF detectors are presented in Section 2. Simulations and expected mass resolutions will be discussed in Section 3.

\section{FALSTAFF experimental apparatus}

- The detection of low energy heavy ions in direct kinematics is difficult since their energy loss is important while they cross materials. These losses have to be taken into account to reconstruct the correct velocity and energy. This implies that the thickness of material layers has to be reduced as much as possible and that the ion positions on the different layers have to be precisely measured to calculate the crossed thickness and then apply the energy loss correction.

- Mass resolution is completely dependent on the time, energy and space resolutions. Desired resolutions are $150 \mathrm{ps}, 1 \%$ and $1.5 \mathrm{~mm}$ for time, energy and space resolutions respectively.

- The flux at a distance of $5 \mathrm{~m}$ from the NFS converter is $1.8 \times 10^{6} \mathrm{n} / \mathrm{cm}^{2} / \mathrm{s} / \mathrm{MeV}$ for an incident deuteron intensity of $50 \mu \mathrm{A}$. A statistics of 1000 events is required for the mass yield of $1 \%$. Then, with a $150 \mu \mathrm{g} / \mathrm{cm}^{2}$ thick target and assuming a $1 \mathrm{MeV}$ bin size for the neutron energy, a geometrical acceptance of about $2 \%$ of $4 \pi$ is needed to keep the beam time shorter than 2 weeks.

Therefore the use of large detectors with low budget material, high performances and allowing position reconstruction is mandatory. The fragment energy will be measured with ionization chambers, this type of detector being able to reach the desired 1\% energy resolution [3]. The fragment nuclear charge identification will be provided by $\Delta \mathrm{E}-\mathrm{E}$ correlation up to $\mathrm{Z}=40$. Among the different types of existing ionization chamber setups, segmented or axial chambers are foreseen. In addition, a more advanced detector, providing the collection of the scintillation photons generated by ions crossing the gas, is under study. This latter solution could provide a fast time signal in addition to the energy measurement. This could allow to get rid of the time-stop detector. Since this innovative R\&D is still in progress, an axial chamber, not be presented here, will be constructed for the first version of the setup.

For the TOF measurement, the necessary timing resolution will be obtained by a combination of an emissive foil and a MWPC (Multi Wire Proportional Counter) as secondary electrons detector (SED) [2]. Large area can be covered with this type of detector without degrading the time and spatial resolution. The most recent results on the R\&D of a MWPC-Sed prototype are presented in the next section.

The first version of the setup will be composed of one arm consisting of two MWPC-Sed detectors together with an existing ionization chamber (MiniHarpee). This setup will be tested with a Cf-252 source in order to measure the final mass of light fragments (the nuclear charge of fragments with $\mathrm{Z}>40$ cannot be identified with a segmented ionization chamber). In a second step, a second arm will be added in order to realize the coincidence $2 \mathrm{~V}$ and $\mathrm{EV}$ techniques (Fig. 1). For this setup, two axial ionization chambers will replace the MiniHarpee. A spectroscopic Cf source, providing the two fission fragments, is presently under development for this setup. This setup will first allow verifying the resolutions obtained for final and initial masses of light and heavy fragments. Then the setup will be moved to NFS for a "day-one" experiment on ${ }^{238} \mathrm{U}$. In a last step, the solid angle of the FALSTAFF setup will be increased by adding two other arms thus allowing the collection of a better statistics or the use of thinner targets.

\subsection{Characterization of MWPC-SED detectors}

An aluminized Mylar foil of $0.9 \mu \mathrm{m}$ thickness, inclined at $45^{\circ}$ with respect to the horizontal direction and polarized at $-10 \mathrm{kV}$, is placed along the path of the fission fragments. A grounded grid placed at $1 \mathrm{~cm}$ from the emissive foil allows having a high electric field close to the foil. The secondary electrons extracted by the crossing ion from the Mylar foil are then accelerated to $10 \mathrm{keV}$ and fly towards the 


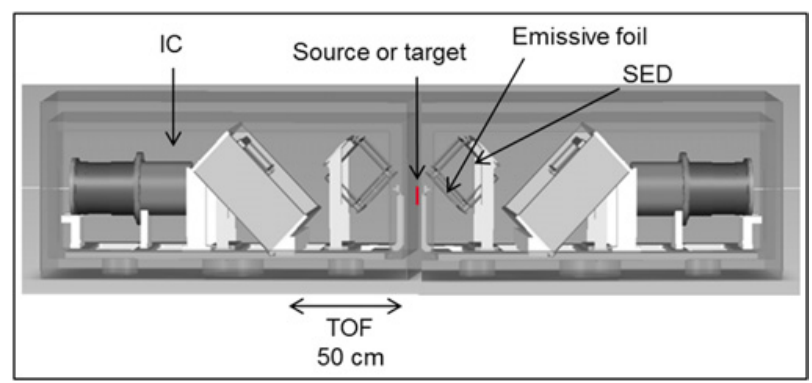

Figure 1. Sketch of the FALSTAFF apparatus.
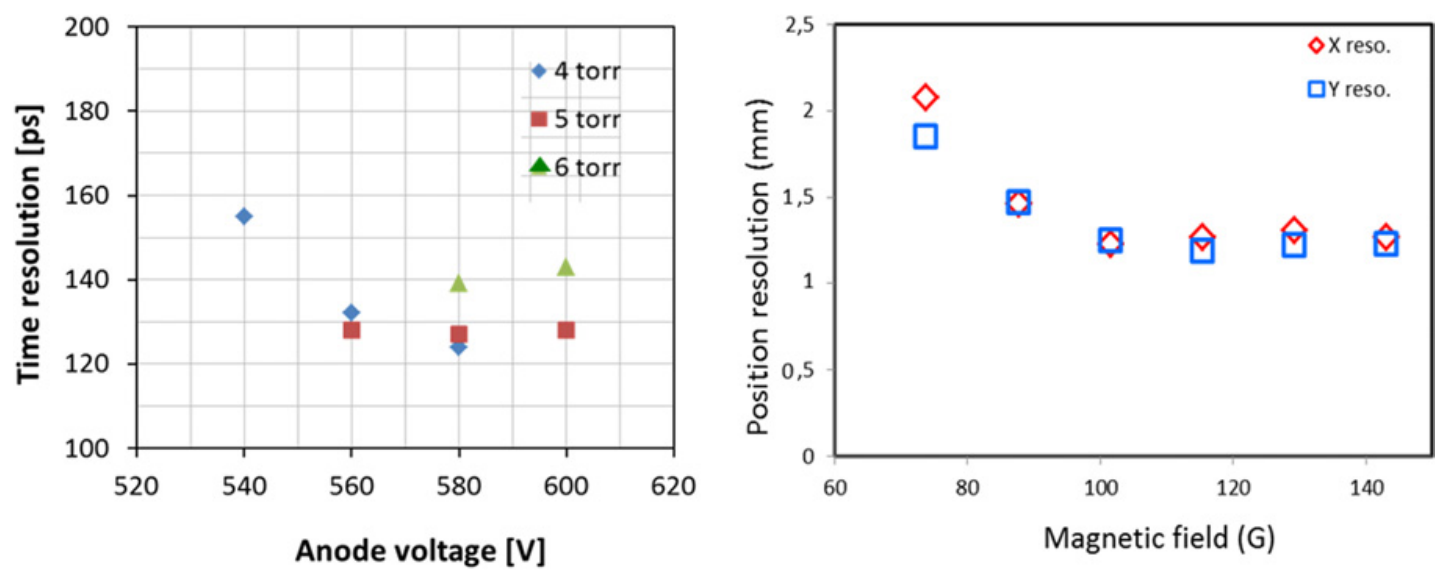

Figure 2. Time resolution as a function of the amplification voltage (left panel) and space resolution as a function of the magnetic field.

MWPC detector. The accelerated electrons pass through the MWPC entrance foil $(0.9 \mu \mathrm{m}$ aluminized Mylar) with $70 \%$ efficiency. They create ionization electrons inside the gas which are then amplified while drifting towards the cathode. The amplification takes place over the whole drift path thanks to the low pressure of the gas (4-6 Torr). The time signal is then read from the wire plane by a fast amplifier. The spatial information is taken from the charge induced on a 2D pixelized cathode.

A MWPC-SED prototype of $15 \mathrm{~cm} \times 20 \mathrm{~cm}$ active area with a pixelized cathode $(68 \times 48$ pads $)$ and a gap of $1.6 \mathrm{~mm}$ between the entrance window and the anode has been built and tested in laboratory with fission fragments of a spectroscopic Cf-252 source. Both time and space resolutions have been measured. The time resolution as a function of the voltage applied between the entrance window and the anode is shown in the left panel of Figure 1. The best obtained resolution is $120 \mathrm{ps}$ but further improvements are expected from noise reduction, possibly improving the resolution to $100 \mathrm{ps}$. The space resolution of the MWPC-SED depends on the focalization of the secondary electrons reaching the SED entrance window. Therefore, the detector is placed inside a dipole providing a field of a hundred of gauss. The right panel of Figure 2 shows the spatial resolution as a function of the magnetic field. A resolution of $1.5 \mathrm{~mm}$ is reached for a field of 120 gauss. The measured time and spatial resolutions already meet the specifications requested from this detector to achieve a satisfactory mass resolution. The actual values of time and space resolutions are used in the simulations of the full setup. 


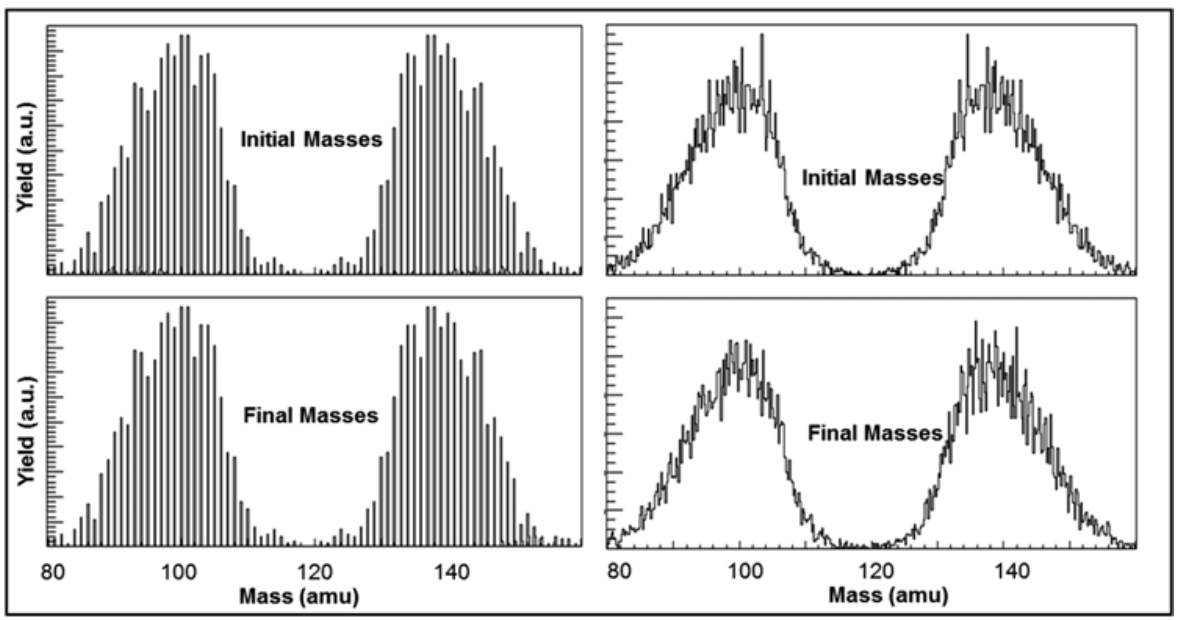

Figure 3. Initial and Final fragment mass distributions with (right panel) and without (left panel) energy straggling and detection resolutions.

\section{Simulations}

A full simulation of the FALSTAFF two-arm setup has been performed in order to develop the analysis method and optimize the achievable mass and charge resolutions taking into account the different layers of material and the space, time and energy resolutions of the different detectors. A detailed geometry of the detection system shown in Figure 2 has been implemented in the GEANT4 Monte-Carlo code. To reconstruct the initial and final mass of both fission fragments from their velocity and energy, the analysis is based on an event-by-event iterative procedure that takes into account the energy lost in the target and the different foils. As an example, the initial and final mass of two typical fission fragments in coincidence (Cs-140 and Rb-98) is correctly reconstructed with a mass resolution better than $0.01 \mathrm{amu}$, coming only from the energy loss correction uncertainty. With the addition of the energy straggling together with the experimental resolutions, we obtain a resolution better than 1 amu for the initial mass and $1.9 \mathrm{amu}(1.6 \mathrm{amu})$ for the heavy (light) fragment final mass. For the whole fission fragment mass distribution, the reconstructed initial and final mass distributions with (without) simulating the energy straggling and the experimental resolutions are shown on the left (right) panel of Figure 3. As expected, the energy straggling and the experimental resolutions have the effect to smear out the reconstructed mass distributions. Nevertheless, including the evaporation process, the feasibility of extracting the neutron multiplicity as a function of the initial mass could be demonstrated. In the analysis of the simulation data, the neutron multiplicity is again determined on an event-by-event basis. Then, for a given initial mass, the average neutron multiplicity $\left(\left\langle A_{i}-A_{f}\right\rangle\right)$ and its uncertainty $\left.\left(\left\langle A_{i}-A_{f}\right\rangle\right) / \sqrt{N}\right)$ are calculated over $N$ events. The reconstructed neutron multiplicity, together with the simulated one, is in Figure 4. One can clearly see that the extracted correlation is very satisfactory. The lack of statistics in the wings and the symmetric valley of the mass distribution lead to larger uncertainties and some gaps between simulated and reconstructed values. We observe also a steeper slope for reconstructed light fragments that has to be investigated further.

\section{One arm setup}

A picture and a sketch of the first version of the setup, composed of one arm, are shown in Figure 5. Tests with a Cf source will begin soon in order to confirm the ability of the setup for the determination of final fission fragment masses. 


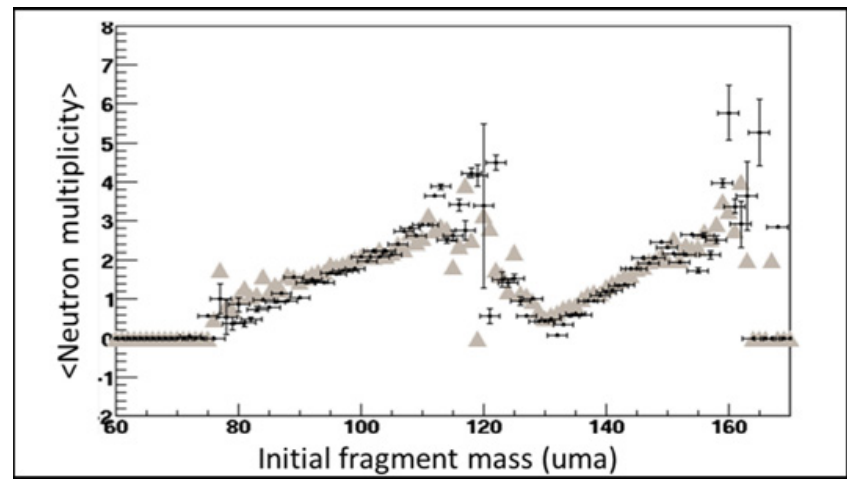

Figure 4. Mean neutron multiplicity as a function of the initial fragment mass. The grey triangles represent the simulated neutron multiplicity and the crosses the reconstructed one.

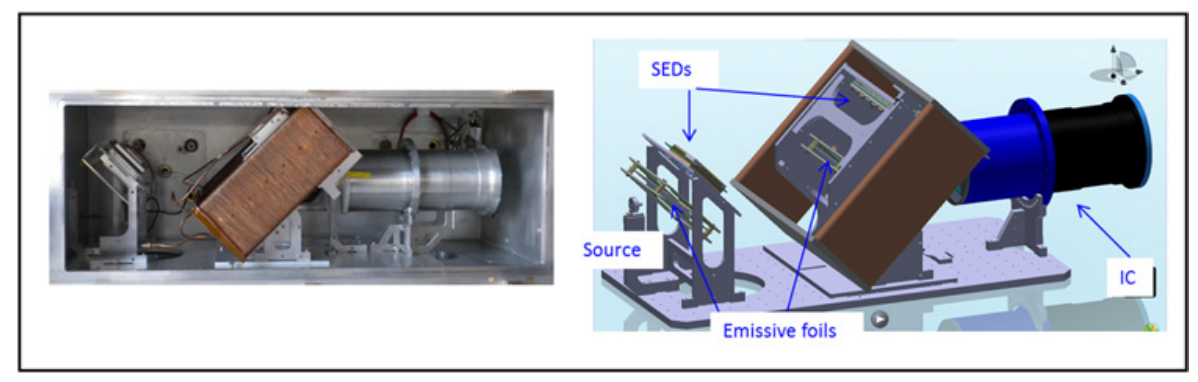

Figure 5. Picture and sketch of the first arm os the FALSTAFF setup.

\section{Conclusions}

A new experimental setup dedicated to the characterization of actinide fission fragments is under development. In addition to the charge, kinetic energy, initial and final mass of both fragments in coincidence, the FALSTAFF setup will allow determining the neutron multiplicity associated to each fragment. A detailed simulation of the apparatus shows that the foreseen experimental setup with already achieved time and space resolutions is able to provide the required precision. The first test with one arm of FALSTAFF is going to be performed in May 2013. The next step will be the addition of the second arm for determination of initial and final mass for light and heavy fragments. Falstaff setup will then be moved to NFS for the first in-beam experiment.

\section{References}

[1] H.C. Britt et al., Instr. AND Meth. 24, 13 (1963)

[2] J. Pancin et al., JINST 4, P120012 (2009)

[3] J.P. Bocquet et al., NIMA 267, 466 (1988) 